\title{
Relaciones verticales y competencia en el mercado español de distribución de carburantes: el impacto de las características de la demanda
}

Alejandro Bello* y Sandra Cavero**

\section{RESUMEN}

Tras el proceso de apertura y liberalización de la industria del petróleo española iniciado en 1992 ha habido cambios notables en las relaciones verticales que se establecen en la industria. Nuestro trabajo se centra en estudiar el impacto de determinadas características de los mercados sobre la demanda y los beneficios de las empresas y su efecto en la probabilidad de encontrar estaciones de demanda y los beneficios de las empresas y su efecto en la probabilidad de encontrar estaciones de
servicio abanderadas frente a estaciones de servicio independientes. Se propone un modelo teórico servicio abanderadas frente a estaciones de servicio independientes. Se propone un modelo teórico
que recoge la estructura competitiva del sector y se analizan a partir de los resultados en el equilibrio las variables que orientan las decisiones de los empresarios. La parte empírica del trabajo analiza dichos factores en el mercado de distribución minorista de carburantes utilizando una muestra de 4,164 estaciones de servicio.

Número de clasificación: JEL: L11, L14, L71.

Palabras clave: Relaciones verticales, abanderamiento, competencia, distribución de carburantes, España.

\section{Abstract}

After the opening and liberalization process of the Spanish oil industry initiated in the last decade, the vertical relationships between refineries and retailers have experienced important changes. Ou study examines the impact of market characteristics upon the demand and upon the profits of the service stations and on the probability to find different service stations according with its brandnames. We propose a theoretic model that represents the competitive structure of the sector and from the equilibrium results we analyze how certain factors affect the contractual elections. The empirical part of the paper tests these factors in the Spanish retail gasoline market using 4,164 empirical part
service stations.

Number of classification: JEL: L11, L14, L71.

Key words: Vertical relationships, brandnames competence, fuels distribution, Spain.

* Profesor en el Departamento de Gestión de Empresas de la Universidad Pública de Navarra, España [alejandro.bello@unavarra.es]

** Profesora titular de Dirección Estratégica en la Universidad Pública de Navarra, España [cavero@ unavarra.es]. 


\section{INTRODUCCIÓN Y REVISIÓN BIBLIOGRÁFICA}

Este trabajo profundiza en el estudio de factores determinantes de la estructura competitiva del sector de distribución minorista de carburantes y combustibles de automotores en España. El estudio de estos aspectos cobra especial relevancia en la actualidad, ya que recientemente, y en especial como consecuencia del proceso de apertura y liberalización de la industria española del petróleo iniciado en 1992, se viene experimentando una serie de cambios en las estrategias de las empresas que están afectando a la estructura competitiva del sector en lo relativo al tipo de estaciones de servicio que operan en el mercado final (Bello y Cavero, 2007a; 2008). El elemento esencial de estos cambios se encuentra en el aumento de flexibilidad y poder de decisión de las estaciones de servicio a la hora de negociar sus contratos con las refinerías, de tal forma que pueden decidir si operan con su respaldo de marca por medio de contratos de abanderamiento en exclusiva o, por el contrario, si lo hacen por su propia cuenta de forma independiente. ${ }^{1}$ Varios cambios han dado impulso a esta nueva situación.

Desde el punto de vista normativo, se han registrado importantes avances hacia la competencia en favor de un incremento en la libertad y el poder de negociación de los empresarios de estaciones de servicio con las refinerías. La reciente entrada en vigor de la normativa europea que limita la duración máxima de los contratos de suministro y venta en exclusiva a cinco años ha supuesto un importante acortamiento de la duración de los contratos, y con esto el empresario de estaciones de servicio tiene mayor flexibilidad para cambiar de operador. ${ }^{2}$ Esta normativa afecta fundamentalmente los contratos DODO y CODO, que constituyen las formas contractuales mayoritarias. Además se ha suprimido la barrera administrativa representada por la normativa que exigía a las nuevas aperturas tener garantizado el abastecimiento por medio de contratos en exclusiva con las refinerías antes de la entrada en funcionamiento del establecimiento. De esta forma, los

${ }^{1}$ El sector de distribución minorista de carburantes en España es muy heterogéneo. En él conviven estaciones de servicio de gran escala, integradas verticalmente o abanderadas en exclusiva con ofreciendo precios bajos y un producto de menor calidad (imagen, marca y servicios). Básicamente
se distinguen cinco tipos de estaciones de servicio en el mercado final, según el contrato que las ligue a las refinerías: coco (Company Owned-Company Operated), codo (Company Owned-Dealer Operated), DoDo (Dealer Owned-Dealer Operated), coDo (Dealer Owned-Company Operated) e independientes (Bello y Cavero, 2007 b).

${ }^{2}$ Hasta el fin del "Monopolio de Petróleos" en 1992, las estaciones de servicio operaban con contratos de suministro y venta en exclusiva con las grandes refinerías nacionales de larga duración que oscilaban entre 10 y 25 años de duración. empresarios de estaciones tienen mayor capacidad negociadora a la hora de optar entre distintos operadores y formas contractuales.

Por otra parte, el proceso de apertura y liberalización ha traído consigo un considerable aumento del número de estaciones de servicio que han pasado de 4,800 en 1992 , a 8,800 en junio de 2005 , siendo el crecimiento sostenido y especialmente intenso en los últimos años. ${ }^{3}$ Este incremento ha estado impulsado básicamente por un aumento considerable de estaciones de servicio independientes ("blancas"). La gráfica 1 muestra la tasa de variación porcentual entre años de los puntos de venta que componen dicha red.

GRÁfICA 1. Tasa de variación porcentual entre años del número

de estaciones de servicio que componen la red de estaciones de servicio

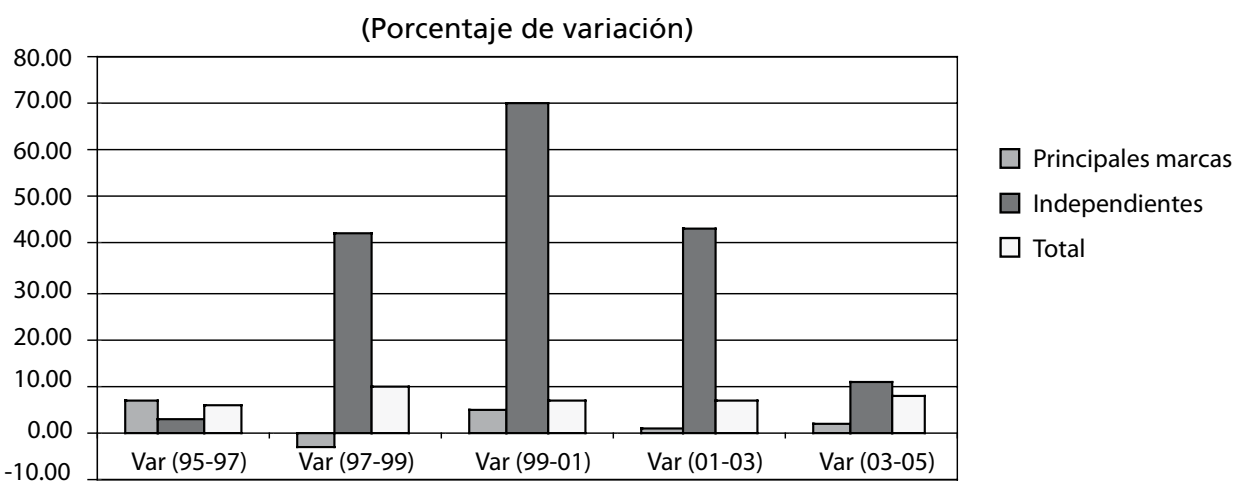

Fuente: Enciclopedia del petróleo, petroquímica y gas. Años: 1995 a 2006. Madrid: ollGAs.

Actualmente, las estaciones de servicio independientes constituyen el tercer grupo de importancia detrás de las reconocidas marcas de Repsol yPF y Cepsa, agrupando a más de 1,250 estaciones de servicio (15 por ciento del total). Muchos contratos de larga duración firmados entre las gasolineras y las refinerías al finalizar el monopolio, así como otros celebrados durante el periodo de transición, están venciendo: un número considerable de gasolineras optan por no renovar sus contratos con las refinerías y pasan a operar de forma independiente o con nuevos distribuidores (Segrelles, 2003-www.aop.es). ${ }^{4}$

${ }^{3}$ El proceso de apertura y liberalización del sector de hidrocarburos en España iniciado en 1992 ha impulsado el ingreso de nuevos operadores extranjeros así como la apertura de nuevas gasolineras por parte de hipermercados y empresarios particulares, especialmente a partir de medidas como el Real Decreto 15/1999 por el que se aprueban las Medidas de Liberalización, Reforma Estructural e Incremento de la Competencia en el Sector de Hidrocarburos, o el Real Decreto 6/2000 de Medidas Urgentes de Intensificación de la Competencia en Mercados de Bienes y Servicios.

${ }^{4}$ Informe de Coyuntura 2003. Jorge Segrelles fue director de la Asociación de Operadores Pe- 
Tanto el aumento global de la red de estaciones de servicio como el aumento de estaciones independientes son elementos determinantes de la intensidad competitiva y los precios finales en el sector, y han sido objeto de análisis en varios estudios recientes. Barron et al. (2004), usando una muestra de 3,000 estaciones de servicio de San Francisco, San Diego, Phoenix y Tucson, ponen de manifiesto que un aumento en el número de estaciones de servicio que compiten en un mercado produce dos efectos en la competencia: por un lado, una disminución de los precios finales y, por otro, una menor dispersión de precios entre gasolineras de una misma región. Por otra parte, otros trabajos dejan constancia de que el incremento en la presencia de estaciones independientes o "blancas" en los mercados determina menores precios como resultado de una intensificación de la competencia (Meerbeeck, 2003; Hastings, 2004), y que generalmente ofrecen un producto de menor calidad (sin marca, sin aditivos registrados y generalmente con un menor nivel de servicios) a un menor precio.

Si bien nuestro estudio se centra en los efectos de las características de la demanda sobre la estructura competitiva, los cambios registrados en el sector de distribución minorista en estaciones de servicio exigen tener en cuenta los importantes resultados que aportan las referencias anteriores. Desafortunadamente no contamos con referencias españolas o europeas que hayan introducido estos aspectos en el análisis de la competencia en este sector.

El aumento de la red de estaciones de servicio trajo consigo una caída de los volúmenes comercializados por cada establecimiento. Según la AOP, en 1990 la media de los volúmenes comercializados por cada estación de servicio era de 4.2 miles de $\mathrm{m}^{3}$, y desde entonces se ha registrado una caída sostenida hasta 1999, cuando los volúmenes se situaban en torno a 3.2 miles de $\mathrm{m}^{3}$. Actualmente, se registra una recuperación en dichos volúmenes, que forma parte de una tendencia creciente, y se sitúan en torno a los 3.4 miles de $\mathrm{m}^{3}$, hecho que pone en evidencia los importantes esfuerzos realizados por los empresarios para encontrar fórmulas y estrategias que permitan rentabilizar el negocio. En este sentido se pueden distinguir dos comportamientos distintos en cuanto a las estrategias seguidas por los operadores. Por un lado, los grupos con mayor prestigio y participación en el mercado final, Repsol YPF, Cepsa y BP, que han sido los más castigados por las regulaciones procompetitivas impulsadas por el gobierno, han centrado sus esfuerzos en ofrecer un producto diferenciado, para lo cual se han realizado importantes esfuerzos en la mejora tecnológica de los productos,

trolíferos en España en 2003. Esta asociación la integran los principales operadores del sector: Agip España, Bp España, Cepsa, Erg Petróleos, Esso Española, Galp Energía, Kuwait Petroleum España, Oilinvest España, Repsol-YPF, Shell España y Total Spain. así como en la promoción de sus marcas de calidad. Además han incorporado distintos servicios que han ampliado el negocio tradicional de las estaciones de servicio: tarjetas de financiación y descuento, tiendas de conveniencia, servicios de hostelería y restauración. Por otro lado, los empresarios independientes han llevado a cabo una estrategia más agresiva, centrada en el volumen comercializado, ofreciendo una gasolina sin marca adquirida en el mercado libre al precio mayorista más conveniente y comercializada, en general, a precios más bajos.

En este contexto, el tipo de contrato que vincula a las estaciones de servicio con los suministradores (refinerías o mayoristas) es un factor determinante para la competencia, dados sus efectos en las estrategias de fijación de precios y diferenciación de producto, que derivan del contrato. Una estación de servicio operará con el respaldo de marca de una refinería de reconocido prestigio en el mercado local, o por el contrario lo hará de forma independiente sin respaldo de marca, en función del tipo de contrato que la relaciona con su suministrador.

Si el empresario de estaciones de servicio decide abanderar su estación de servicio con la marca de una refinería reconocida en el mercado local, puede obtener varios beneficios; en primer lugar, el derivado del respaldo de la marca reconocida. Según PNG y Reitman (1995), los consumidores encuentran en la marca una verdadera y creíble señal de calidad superior que permite aumentar los precios sin disminuir el excedente del consumidor. Además, la afiliación a una compañía petrolera permite al empresario no asumir el riesgo de las fuertes oscilaciones en el precio del crudo, que en el caso de que el carburante se adquiera en el mercado libre, se transmiten de forma inmediata en los precios mayoristas y de éstos a los precios finales. Por último, el gestor de la estación de servicio puede disfrutar de otros beneficios derivados de la gestión del negocio: apoyo en la gestión y el merchandising, formación, promociones, etcétera.

Sin embargo, la afiliación de una estación de servicio también implica ciertos perjuicios para el empresario de dicha estación. En primer lugar, generalmente supone costos mayores, ya que para obtener el respaldo de la refinería generalmente debe pagar un canon fijo, con lo que el precio mayorista suele ser mayor que el precio de libre mercado. Además, la estación de servicio afiliada a una bandera, tanto con un contrato CODO o DOCO, debe cumplir con algunos requisitos operativos impuestos por el operador, como imagen, servicios de atención al cliente y mantenimiento de instalaciones, horarios, etc., que también pueden suponer un costo mayor que el que soportan las estaciones de servicio independientes. En segundo lugar, la reputación que se genera implica un mayor costo para una estación de servicio que decida cambiar de operador, ya que pierde parte de la reputación que haya adquirido hasta entonces. Por último, están las posibles 
restricciones sobre la fijación de precios finales. En el caso de los contratos como comisionista, sobre el precio mayorista se fija una comisión de la cual el empresario de la estación de servicio obtiene sus beneficios brutos. Es decir, la decisión del precio corre por cuenta de la refinería. No obstante, en determinadas circunstancias el empresario puede bajar los precios asumiendo tal rebaja en detrimento de sus beneficios, aunque en la práctica existen muy pocos incentivos para bajar los precios en estas condiciones.

Dado que en el mercado final de distribución en España conviven distintos tipos de estaciones de servicio en las diferentes regiones, nuestro trabajo tiene como objetivo estudiar en qué condiciones de mercado la afiliación de una estación de servicio a una marca reconocida puede suponer ingresos lo suficientemente altos como para hacer frente a los costos que supone operar de forma independiente. Nuestro trabajo pretende arrojar luz sobre esta cuestión, analizando el efecto que las características del mercado, principalmente el nivel de ingresos y la densidad de la demanda, tienen sobre la estructura competitiva del sector, mediante su efecto en la demanda y los beneficios de las empresas.

En la bibliografía especializada del sector no encontramos trabajos que valoren el impacto de las características de la demanda sobre la estructura competitiva en el sector de distribución de carburantes y combustibles de automotores en estaciones de servicio. ${ }^{5}$ La atención se ha centrado más en estudiar la estructura competitiva del sector desde dos frentes. Por un lado, existe una amplia bibliografía, principalmente estadounidense, que analiza la elección de contratos en este sector desde un punto de vista de eficiencia asociado a la presencia de costos de agencia (Barron y Umbeck, 1984; Slade, 1993; Blass y Carlton, 2001). Por otro lado están los trabajos antes mencionados que analizan el impacto del número de competidores en los precios finales (Meerbeeck, 2003; Barron et al., 2004).

Si bien el impacto de las características de la demanda sobre la estructura competitiva que queremos estudiar en nuestro trabajo no ha sido analizado en profundidad en este sector, sí existen trabajos que incorporan estos aspectos para controlar sus posibles efectos sobre otros temas concretos. G. Iyer y P. Seetharaman (2003) investigan la decisión de una estación de servicio de discriminar precios entre distintos consumidores en un mercado, utilizando un modelo de

${ }^{5}$ Empíricamente, existen algunos trabajos que analizan la elección de contratos en este sector desde un punto de vista de eficiencia asociado a la presencia de costos de agencia. Cuando la razón capital/trabajo es alta (baja) y los servicios no observables bajos (altos), la solución de integración vertical (separación vertical) es la preferida por las refinerías (Barron y Umbeck, 1984, para Maryland; Slade, 1993, para Vancouver; Blass y Carlton, 2001, para Massachusetts; Hastings, 2004, para California). decisión que se contrasta por medio de una encuesta con información de precios de 198 estaciones de servicio y las características socioeconómicas de los mercados en los que operan, de forma similar al trabajo de S. Borenstein (1991). A. Eckert y W. Douglas (2005) incorporan las características de los mercados para describir el modelo de competencia y fijación de precios, utilizando una muestra de estaciones de servicio de Vancouver. Por otra parte, Barron et al. (2000) analizan las diferencias de márgenes en la comercialización de distintos tipos de gasolinas ("regular" o "premium") entre distintos tipos de estaciones de servicio asociadas a la distancia entre ellas y a las características socioeconómicas de los clientes potenciales. ${ }^{6}$

Los resultados del modelo permiten extraer dos conclusiones importantes acerca del impacto de las características de la demanda sobre la competencia. En primer lugar, al aumentar la densidad de población, aumentan la demanda y los beneficios de las empresas, y lo hacen en mayor proporción en las estaciones de servicio abanderadas que en las independientes. En segundo lugar, la demanda y los beneficios de las empresas aumentan con un incremento en el nivel de ingresos. Este incremento es mayor para las estaciones de servicio que operan abanderadas con marcas reconocidas que para aquellas que operan independientemente.

Empíricamente, nuestro trabajo se centra en el efecto de las características de la demanda de los mercados sobre la probabilidad de encontrar estaciones de servicio abanderadas con marcas reconocidas o independientes. Hemos utilizado una muestra de 4,164 estaciones de servicio perfectamente identificadas por sus marcas, correspondientes a 22 provincias españolas. Esto representa una muestra muy heterogénea en cuanto a las estaciones de servicio, así como a los mercados en los que operan. Los resultados indican que la probabilidad de encontrar estaciones de servicio abanderadas con marcas reconocidas se incrementa con mayores niveles de ingreso y densidad de población.

El trabajo se organiza como sigue: a continuación se presenta el modelo teórico propuesto para analizar los beneficios y a partir del cual se hacen algunas proposiciones. Después se describe brevemente la situación actual del sector, así como los datos con los que se realizará el contraste empírico. Finalmente se presentan las conclusiones finales.

\footnotetext{
${ }^{6}$ Las características de la demanda también se han tenido en cuenta en estudios de regulación y competencia: Vita (2000) estudia el impacto sobre la estructura competitiva del divorcement law en el mercado estadounidense; Comanor y Riddle (2003) analizan la reciente regulación en California ("The Open Branded Supply") y sus efectos sobre la competencia.
} 


\section{MODELO TEÓRICO}

\section{Supuestos generales}

Considérese una estructura vertical constituida por dos refinerías, $R_{a}$ y $R_{b}$, y dos estaciones de servicio, $E_{a}$ y $E_{b}$. Las empresas se diferencian por la calidad del producto que ofrecen y se denotan con el subíndice $a$ (para alta calidad) y $b$ (para baja calidad).

Las refinerías incurren en un costo marginal $c$ y unos costos fijos (y hundidos) que soportan la mejora tecnológica del producto, así como el apoyo publicitario a la marca e imagen del mismo.?

La estación de servicio $E_{a}$, que opera con la bandera de una refinería de reconocido prestigio, compra $d_{a}$ unidades del producto de alta calidad que vende la refinería $R_{a}$ a un precio mayorista $W_{a}$, y las vende en el mercado final a un precio final $P_{a^{\prime}}$. Por otra parte, la estación de servicio $E_{b^{\prime}}$ que es una gasolinera independiente, adquiere $d_{b}$ unidades de carburante de otra refinería (o mayorista) de menor calidad $R_{b}$ a un precio mayorista $W_{b}$, y las comercializa a un precio unitario $P_{b}$ en el mercado final. Utilizamos precios lineales. ${ }^{8}$

Todas las empresas maximizan sus beneficios de tal forma que:

$$
\begin{aligned}
& \operatorname{Max}_{w_{a}} \pi R_{a}=\left(w_{a}-c\right) d_{a} \\
& \operatorname{Max} \pi R_{b}=\left(w_{b}-c\right) d_{b} \\
& w_{b} \\
& \operatorname{Max} \pi E_{a}=\left(p_{a}-w\right) d_{a} \\
& p_{a} \\
& \operatorname{Max} \pi E_{b}=\left(p_{b}-w\right) d_{b} \\
& p_{b}
\end{aligned}
$$

\footnotetext{
${ }^{7}$ La refinería de alta calidad incurriría en mayores costos fijos, mientras que los costos marginales no necesariamente difieren entre las empresas.

${ }^{8}$ Esto trae consigo el problema de la doble marginalización, como consecuencia de que los integrantes del canal distorsionan el precio (lo aumentan) al añadir su propio margen en cada etapa de producción, con la consecuente disminución de la demanda (Spengler, 1950). No obstante, la ineficiencia que se produce como consecuencia de este doble margen es contrarrestada, en parte, con la posibilidad que tienen las estaciones independientes de adquirir el carburante de forma libre en el mercado y comercializarlo a un menor precio (Mills, 1995).
}

Formalmente, el proceso de fijación de precios se describe como un juego en dos etapas con cuatro jugadores. En la primera etapa, la refinería $R_{a}$ decide el precio $W_{a}$ que cobra a la estación de servicio $E_{a^{\prime}}$ mientras que $R_{b}$ hace lo mismo con $E_{b}$. En una segunda etapa, una vez fijados los precios mayoristas, $W_{a}$ y $W_{b}$ por parte de $R_{a}$ y $R_{b}$, respectivamente en la etapa anterior, las estaciones de servicio $E$ y $E_{b}$, deciden de manera simultánea y no cooperativa los precios finales de venta del producto, $P_{a}$ y $P_{b}$.

Por otra parte, los consumidores se encuentran distribuidos en el espacio en función de sus ingresos entre un nivel máximo de ingresos $I_{h}$ y un nivel mínimo de ingresos $I_{l}$. Se asume una función de densidad $f(I)$ unitaria y uniforme en el intervalo $\left(I_{h}, I_{l}\right)$, y $I_{h}+1$. Asumimos que el mercado está cubierto, es decir, que no hay salida de consumidores. Los consumidores compran al menos una unidad del bien a la estación de servicio que ofrezca el producto con la mejor combinación calidad-precio. La utilidad de un consumidor con un nivel de ingresos $I \varepsilon\left[I_{h} I_{l}\right]$ es (véase la figura 1):

$$
U(I)=\left(I-p_{a}\right) s_{a}
$$

si compra una unidad a $E_{a}$, y

$$
U(I)=\left(I-p_{b}\right) s_{b}
$$

si compra una unidad a $E_{b}$.

Si no se realiza ninguna compra, la utilidad es normalizada a cero.

$s_{i}$, representa la calidad $s$ de la empresa $i, s_{i} \varepsilon\left[s_{a}, s_{b}\right]$, siendo $s_{a} \geq s_{b}$.

FIGURA 1.

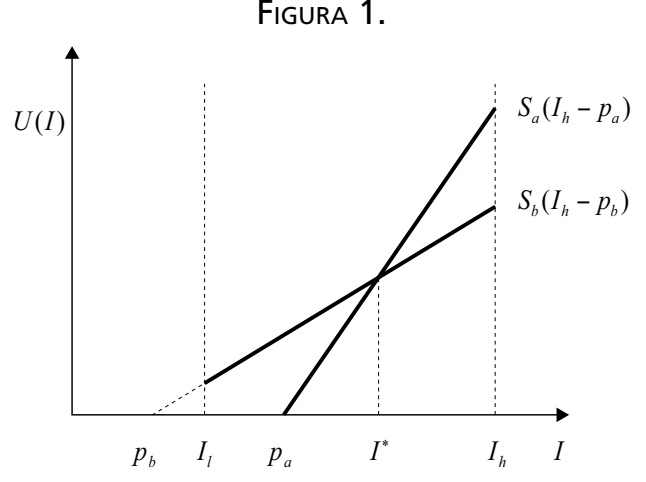


En este contexto se realizan las siguientes hipótesis:

- $0<I_{l}<4+c$, lo que permite que las dos variedades puedan encontrar una demanda positiva en el equilibrio.

- No existen diferencias en cuanto a la apreciación de la calidad del producto por parte de los consumidores.

- Los costos de búsqueda para el consumidor son iguales a cero.

- La demanda total es rígida, normalizada a 1 y se reparte entre las empresas dependiendo de los precios y las calidades.

La posición relativa al nivel de ingresos de los consumidores indiferentes entre las dos variedades del producto determina las funciones de demanda. Un consumidor cuyo nivel de ingresos sea $I$, estará indiferente entre comprar el carburante de alta calidad y el de baja calidad si $\left(I-p_{a}\right) s_{a}=\left(I-p_{b}\right) s_{b}$. A partir de esta relación se determinan las funciones de demanda de las dos variedades, que dependerá del nivel de renta, el precio y la calidad del producto, y la densidad de consumidores $d:^{9}$

$$
\begin{gathered}
q_{a}=\left(I_{h}-\frac{p_{a} s_{a}-p_{b} s_{b}}{s_{a}-s_{b}}\right) d \\
q_{a}=\left(\frac{p_{a} s_{a}-p_{b} s_{b}}{s_{a}-s_{b}}\right) d .
\end{gathered}
$$

\section{Determinación del equilibrio}

El juego planteado se resuelve por inducción hacia atrás. En primer lugar se resuelve la segunda etapa; es decir, las estaciones de servicio, $E_{a}$ y $E_{b}$, reaccionan a los precios mayoristas fijados por las refinerías y maximizan (3) y (4). Consideramos que el grado de diferenciación es $\alpha=\frac{s_{b}}{s_{a}}$, donde $0 \leq \alpha<1$, siendo que un pequeño valor de $\alpha$ implica un alto grado de diferenciación y, por tanto, una baja sustitución entre las variedades. Una vez realizadas las sustituciones se obtienen las siguientes funciones de reacción:

$$
p_{a}(w)=\frac{I_{l}(1-\alpha)+2 w_{a}+a w_{b}+2(1-\alpha)}{3}
$$

\footnotetext{
${ }^{9}$ Considerando que $I_{h}>I_{l}$, e $I_{h}>\left(5 I_{l}-c\right) / 4$, lo que asegura una cobertura total del mercado y una demanda positiva de ambas variedades.
}

$$
p_{b}(w)=\frac{-2 I_{l}(1-\alpha)+w_{a}+2 \alpha w_{b}+1-\alpha}{3 \alpha} .
$$

Anticipando las reacciones de las estaciones de servicio (9) y (10) y sus efectos en las cantidades demandadas, se obtiene:

$$
\begin{aligned}
& w_{a}^{*}=\frac{I_{l}(1-\alpha)+c(2+\alpha)+5(1-\alpha)}{3} \\
& w_{b}^{*}=\frac{I_{l}(1-\alpha)+c(1+2 \alpha)+4(1-\alpha)}{3 \alpha} .
\end{aligned}
$$

Sustituyendo, en las funciones de reacción de las estaciones de servicio a los precios mayoristas (9) y (10), se obtienen los siguientes resultados en el equilibrio:

$$
\begin{gathered}
p_{a}^{*}=\frac{4 I_{l}(1-\alpha)+c(5+4 \alpha)+20(1-\alpha)}{9} \\
p_{b}^{*}=\frac{4 I_{l}(1-\alpha)+c(4+5 \alpha)+16(1-\alpha)}{9 \alpha} \\
q_{a}^{*}=\frac{1}{9} d\left(I_{l}-c+5\right) \\
q_{b}^{*}=\frac{1}{9} d\left(I_{l}-c+4\right) \\
\pi E_{a}^{*}=\frac{1}{81} d(1-\alpha)\left(4 I_{l}-c+5\right)^{2} \\
\pi E_{b}^{*}=\frac{1}{81 \alpha} d(1-\alpha)\left(-I_{l}-c+4\right)^{2}
\end{gathered}
$$

Los precios de las variedades en el equilibrio se corresponden con los esperados. En primer lugar, se verifica que el precio de la variedad de alta calidad, $p_{a}{ }^{*}$, es mayor al precio de baja calidad, $p_{b}{ }^{*}, \forall I(13),(14)$. Además, los precios de las dos variedades son crecientes con el grado de diferenciación, ya que tanto $p_{a}{ }^{*}$ como $p_{b}{ }^{*}$ crecen en la medida que la sustitución entre las variedades $\alpha$ es menor y 
por tanto la diferenciación entre las mismas, mayor. Estos resultados se sitúan en línea con los obtenidos en trabajos anteriores (Bello y Cavero, 2007 b).

Además se observa que el nivel de ingreso afecta de forma distinta a los precios de las variedades. En la medida que el nivel mínimo de renta aumenta, el precio de la variedad de alta calidad también aumenta:

$$
\frac{\delta p_{a}}{\delta I_{l}}=\frac{1}{9}(1-\alpha)
$$

y de mayor forma en la medida que aumente el grado de diferenciación entre las variedades. Sin embargo, en la medida que aumenta el nivel mínimo de renta, el precio de la variedad de baja calidad disminuye:

$$
\frac{\delta p_{b}}{\delta I_{l}}=-\frac{4}{9 \alpha}(1-\alpha)
$$

Podríamos pensar que en la medida que el nivel mínimo de renta de una región aumenta, las preferencias de los consumidores se tornan a favor de consumir la variedad de mayor calidad, con lo que la variedad de menor calidad intensifica la competencia en precios: éstos se reducen para no perder clientes. Además, estas diferencias se acentúan más con el grado de diferenciación entre las variedades. En la medida que la diferenciación es mayor y, por tanto, la sustitución menor, el precio de la variedad de alta calidad aumenta mientras que el de baja disminuye.

Las demandas y los beneficios de las empresas en el equilibrio permiten extraer conclusiones importantes acerca de las características del mercado que pueden ser determinantes de la estructura competitiva de los mercados analizados en (15), (16), (17), (18).

El análisis de los resultados en el equilibrio pone de manifiesto que tanto la demanda como los beneficios de las empresas se ven afectados por la variación de los niveles de renta de una región. ${ }^{10}$ En primer lugar, el análisis de la demanda indica que la demanda por la variedad de alta calidad crece conforme aumenta el nivel mínimo de renta $I_{i}$ :

$$
\frac{\delta q_{a}}{\delta I_{l}}=\frac{d}{9}>0
$$

en detrimento de la demanda por el producto de baja calidad,

${ }^{10}$ En el desarrollo, el modelo teórico ha sido simplificado utilizando únicamente el nivel inferior de renta de los consumidores, y dada la función de densidad de distribución de los consumidores, en la medida que aumenta el nivel de renta mínima se produce un incremento global de renta, media y máxima.

$$
y \frac{\delta q_{b}}{\delta I_{l}}=-\frac{d}{9}<0
$$

El resultado es el esperado, ya que en la medida que los consumidores incrementan su nivel de renta tienen mayor poder adquisitivo que les permite adquirir aquellos productos de mayor calidad a un mayor precio, en respuesta al incremento de sus preferencias por la calidad.

En esta línea, el beneficio de la estación de servicio de alta calidad se incrementa conforme aumenta el nivel mínimo de renta de la región, ya que:

$$
\frac{\delta \pi E_{a}}{\delta I_{l}}=\frac{2}{81} d(1-\alpha)\left(I_{l}-c+5\right)>0,
$$

en tanto el beneficio de la estación de servicio de baja calidad baja en estas condiciones:

$$
\frac{\delta \pi E_{b}}{\delta I_{l}}=\frac{2}{81 \alpha} d(1-\alpha)\left(I_{l}-c-4\right)<0 .
$$

Podríamos pensar que en mercados donde los niveles de renta son altos, la probabilidad de que existan estaciones de servicio abanderadas con la marca de refinerías de reconocido prestigio, que se identifican por su calidad superior a partir de las fuertes inversiones en la mejora tecnológica de sus productos, así como en la promoción de su marca e imagen, será mayor. En estas condiciones, la demanda por este tipo de productos y, por tanto, los beneficios de las empresas que lo comercializan, en este caso, estaciones abanderadas con marcas de reconocido prestigio, crecen de forma pronunciada en detrimento de la demanda por el producto de baja calidad.

Proposición 1. El incremento en el nivel mínimo de ingresos en una región tiene un impacto positivo en la demanda y en los beneficios de las empresas. Este impacto es mayor para la demanda y los beneficios de la empresa de alta calidad.

Otra característica del mercado que condiciona la demanda de ambas variedades y los beneficios de las empresas que lo comercializan es la densidad de población. Por un lado, las demandas de ambas variedades presentan el mismo comportamiento con la densidad de población $d$, ya que tanto la demanda del producto de alta calidad $q_{b}$ como la demanda del producto de baja calidad $q_{a}$ aumentan al incrementarse la densidad de población $d$. Se verifica que:

$$
\frac{\delta q_{a}}{\delta d}=\frac{1}{9}\left(I_{l}-c+5\right)>0,
$$


mientras que:

$$
\frac{\delta q_{b}}{\delta d}=\frac{1}{9}\left(-I_{l}+c+4\right)>0 .
$$

A priori, una alta densidad demográfica determina una alta concentración de estaciones de servicio por unidad de superficie, con la consecuente intensificación de la competencia entre estaciones de servicio debido a la ausencia de diferenciación espacial, en línea con Barron et al. (2004). Estas condiciones pueden dar origen a distintos comportamientos estratégicos por parte de los empresarios de estaciones de servicio. Por un lado, una estación podría rentabilizar su actividad diferenciándose de sus competidores, operando respaldada con la marca imagen y servicios de una refinería reconocida, ya que la mayor concentración de estaciones de servicio tiende a aumentar la competencia en precios. Por otro, la posible intensificación de la competencia en precios también podría inducir a las estaciones de servicio a operar de forma independiente, ofreciendo un producto no diferenciado, ya que de esta forma, como señalamos anteriormente, la estación puede fijar con libertad los precios y tiene más margen para adquirir el carburante en el mercado mayorista al precio más bajo, y así centrar su competencia en precios.

No obstante, el aumento de la demanda al aumentar la densidad de población afecta de forma más pronunciada a la variedad de alta calidad, ya que:

$$
\frac{\delta q_{a}}{\delta d}>\frac{\delta q_{b}}{\delta d}
$$

Como es de esperar, los resultados en el equilibrio indican que las empresas se benefician con un incremento de la densidad de población, en tanto se incrementa el volumen comercializado. El beneficio de la estación de servicio de alta calidad aumenta con la densidad de población de forma más pronunciada que el beneficio de la estación de servicio de baja calidad:

$$
\frac{\delta \pi E_{a}}{\delta d}=\frac{1}{81}(1-\alpha)\left(I_{l}-c+5\right)^{2}>\frac{\delta \pi E_{b}}{\delta d}=\frac{1}{81 \alpha} d(1-\alpha)\left(I_{l}-c-4\right)^{2}
$$

Proposición 2. La demanda y los beneficios de las empresas aumentan con la densidad de población. La demanda y los beneficios de la empresa de alta calidad crecen al aumentar la densidad de población en mayor proporción que los del producto de baja.

\section{ANáLISIS EMPírico}

El objetivo de esta parte del trabajo es analizar empíricamente los dos aspectos fundamentales que se estudian en el apartado teórico: el impacto del nivel de ingresos y la densidad de población sobre la estructura competitiva del sector de distribución minorista de carburantes y combustibles de automotores. Centraremos el análisis en estudiar la probabilidad de que una estación de servicio opere bajo el amparo de una marca de reconocido prestigio o, por el contrario, lo haga de forma independiente, en función de las características de la demanda en las distintas regiones españolas.

La base de datos utilizada está constituida por 4,164 estaciones de servicio correspondientes a 22 provincias españolas: Asturias, Badajoz, Baleares, Barcelona, Burgos, Cantabria, Castellón, C. Real, La Coruña, Cuenca, Guipúzcoa, Jaén, Madrid, Málaga, Murcia, Navarra, Orense, Salamanca, Soria, Valencia, Valladolid y Zaragoza. ${ }^{11}$ Las estaciones de servicio han sido localizadas utilizando el siguiente procedimiento: en primer lugar hemos localizado e identificado las estaciones de servicio de la base de datos del Ministerio de Economía, donde se recogen y publican semanalmente los precios de venta de los carburantes de aproximadamente 90 por ciento de las estaciones de servicio de toda España (http://www6. mityc.es/energia/hidrocarburos/carburantes/index.asp). Posteriormente, utilizando la Guía de estaciones de servicio, proveedores y operadores (2003), procedemos a identificar cada una de las estaciones de servicio con su respectivo operador. Además, en dicha publicación obtenemos información referente a la localización marca, servicios, etcétera, que se ofrecen en cada establecimiento.

Se ha recogido la siguiente información para cada una de las estaciones de servicio: precio de venta de la gasolina súper sin plomo de 95 octanos, proveniente de los precios publicados semanalmente por el ministerio de Economía, y marca que abandera cada una de las estaciones de servicio. Adicionalmente, hemos completado la base con información demográfica y socioeconómica de cada uno de los mercados donde operan las estaciones de servicio proveniente de varias fuentes: Instituto Nacional de Estadística: Anuario Estadístico 2003; Boletines mensuales de estadística (2003); Anuario económico y social-La Caixa, Encuesta de Población Activa (EPA), Dirección General de Tráfico (DGT), Informes Económicos Regionales.

${ }^{11}$ Puede observarse que todas las comunidades autónomas del territorio español están representadas al menos por una provincia. 


\section{Medidas empíricas}

De acuerdo con nuestro objetivo, que es analizar la probabilidad de que en algunos mercados predominen estaciones de servicio con respaldo de marca frente a estaciones de servicio independientes, en función de las características de los mercados en los que operan, hemos construido una variable dummi-brand. Esta variable toma valor 1 cuando la estación de servicio opera con una marca de reconocido prestigio y 0 cuando lo hace de forma independiente. ${ }^{12} \mathrm{Hemos}$ considerado que tanto la presencia en el mercado como los esfuerzos en diferenciación de marca e imagen son determinantes para que una estación de servicio pueda ser percibida y reconocida por los consumidores como de mayor calidad. Los grandes operadores destinan importantes sumas a inversiones en publicidad así como en la remodelación de sus estaciones de servicio al incorporar nuevas prestaciones, mientras que las gasolineras independientes o blancas no realizan tales esfuerzos. Por este motivo, debido a que existen marcas con alta presencia nacional (Repsol-YpF, Cepsa, BP) y marcas menores en el nivel nacional pero que son fuertes en mercados regionales - Petrocat (Cataluña), Avanti (País Vasco), Galp, entre otras - en algunos mercados éstas son consideradas como de mayor calidad percibida, mientras que en otros en los que su participación es menor a 3 por ciento son consideradas como de baja. Las variables explicativas utilizadas para el contraste son las siguientes: nivel de ingresos (INGRESOS), densidad de consumidores (DENCONSUM) y densidad de vehículos (DENSVEHICULOS).

En relación con el nivel de ingresos característico en el mercado donde opera la estación de servicio, al igual que en el apartado teórico, hemos utilizado el mínimo nivel de ingresos en la región (MININGRESOS). La variable se obtiene haciendo la media del nivel económico de 10 por ciento de la población total con menores ingresos de cada región. Hemos creado esta variable a partir de los datos del Anuario Económico de la Caixa 2004, utilizando la base de datos con detalle municipal, de donde se obtiene el índice de nivel económico. ${ }^{13}$

${ }^{12}$ Las marcas reconocidas que abanderan las estaciones de servicio en el mercado español son: Repsol-YPF, Cepsa, Bp y, en función de su participación en el mercado, Shell, Agip, Avanti, Avia, Essergui, Galp, Tamoil, Meroil, Petrocat, etc., mientras que las sin marca son las independientes.

Essergui, Galp, Tamoil, Meroil, Petrocat, etc., mientras que las sin marca son las independientes.
${ }^{13}$ Índice de la renta familiar disponible por habitante estimada por áreas geográficas (nivel municipal, provincial y por comunidades autónomas, distribuidos en 10 niveles). La renta personal disponible se puede definir como el nivel de renta de que disponen las economías domésticas para gastar y ahorrar, o bien como la suma de todos los ingresos efectivamente percibidos por las economía domésticas durante un periodo. Por ello, podría considerarse como el total de ingresos procedentes del trabajo, más las rentas de capital, prestaciones sociales y transferencias, menos los impuestos directos pagados por las familias y las cuotas pagadas a la seguridad social.
Para analizar el impacto de la densidad sobre la estructura competitiva hemos utilizado dos variables: DENSITY mide la densidad de población, y es el resultado de dividir la población al 1 de enero de 2003 entre la extensión en kilómetros cuadrados correspondiente a 1996 (último Nomenclátor del INE que facilita esta información). Del mismo modo, la densidad de vehículos se recoge con las variables CAR.

Por otra parte, hemos incorporado al modelo tres variables de control, con el objetivo de recuperar en el modelo los posibles efectos de la localización de la estación de servicio sobre la competencia. Estas variables son: la intensidad de la competencia, medida por la densidad de estaciones de servicio (INTENSIDAD); el tipo de vía en la cual opera la estación de servicio (VIAS), y la presencia de instalaciones de refino y logísticas en la región donde opera la estación (REFINO).

A partir de las aportaciones de Meerbeeck (2003), Barron et al. (2004) y Hastings (2004) sabemos que la densidad de estaciones de servicio puede tener importantes efectos sobre la competencia, la fijación de precios y la estructura competitiva del mercado. Un incremento de la intensidad de la competencia produce una reducción en los volúmenes comercializados y una reducción en los precios finales. El efecto sobre la decisión del tipo de contrato, a priori, es ambiguo, ya que los empresarios pueden optar por distintas alternativas para competir, ya sea diferenciándose por medio del abanderamiento con una marca reconocida o bien centrarse en una estrategia más competitiva en precios.

La variable ROAD, que recoge el tipo de vía en que se ubica la estación de servicio, también puede tener efectos sobre la competencia y la probabilidad de elección de contratos; en vías no urbanas (carreteras y autopistas) se constatan condiciones competitivas singulares debido a que son vías rápidas que desincentivan al automovilista a realizar recargas. Generalmente presentan precios mayores que las estaciones localizadas en centros urbanos. Para efectos de nuestro estudio, en principio sería lógico pensar que una estación de servicio localizada en una vía rápida se beneficiaría abanderándose con una marca reconocida, fácilmente distinguible por los automovilistas que circulen por ésta.

Por último, la variable REFINO recoge la presencia o ausencia de instalaciones de refino y almacenamiento en la provincia. Por medio de esta variable capturamos el efecto que puede tener en la elección de contratos la presencia de una refinería en la provincia en la cual se ubica la estación de servicio. En España, las tres compañías dedicadas al refino están integradas verticalmente y controlan también gran parte de las instalaciones logísticas de almacenamiento (Compañía Logística de Hidrocarburos). Tradicionalmente estas empresas se han especializado en el mercado "aguas abajo" y poseen las marcas con mayor prestigio en el mercado local. A priori, es de esperar que una estación de servicio se beneficie 
doblemente operando con la bandera de una empresa reconocida en la región, por el reconocimiento de la marca en el mercado local y por los menores costos de distribución dada la cercanía de las refinerías y las instalaciones de almacenamiento.

\section{a) Estimación del modelo}

El modelo que proponemos para analizar la estructura competitiva del sector de distribución minorista de hidrocarburos para las distintas provincias españolas es un modelo de regresión logística binomial. Por medio de éste pretendemos analizar la probabilidad de que una estación de servicio represente la marca e imagen de una refinería de prestigio o, por el contrario, sea una estación de servicio independiente, en función de las características socioeconómicas y la intensidad competitiva en el mercado en el que opere. La variable dependiente será BRAND, y las variables PREDICTORIAS, MININGRESOS, DENSCONSUM, DENSVEHICULOS, INTENSIDAD, VIAS Y REFINO.

\section{b) Contraste empírico}

El modelo propuesto para contrastar las proposiciones teóricas es el siguiente:

$B R A N D=\frac{1}{1+e-\left(b_{0}+b_{1} \text { MININGRESO }+b_{2} \text { DENSCONSUM }+b_{3} \text { DENSVEHICULOS }+b_{4} \text { INTENSIDAD }+b_{5} \text { VIAS }+b_{6} \text { REFINO }\right)}$

Donde:

- BRAND: Variable dependiente, dicotómica, que toma valor 1 cuando la estación de servicio está abanderada con la marca e imagen de una refinería de prestigio, y 0 cuando la estación de servicio es una estación de servicio independiente o "blanca".

- MININGRESOS: Variable continua, que recoge la media de los ingresos más bajos de la región. Se distribuye desde el nivel mínimo de 1 hasta 5.71 .

- DENSCONSUM: Variable categórica, que representa la densidad de población de la región. Se distribuye en tres clases: Clase 1: Low; Clase 2: Medium; Clase 3: High.

- DENSVEHICULOS: Variable categórica, que representa la densidad de vehículos de una provincia. Clase 1: Low; Clase 2: Medium; Clase 3 . High.
- INTENSIDAD: Variable dummy, que representa la intensidad competitiva en una provincia. Toma valor 1 cuando la concentración de estaciones de servicio se sitúa por encima de la media nacional y 0 cuando se sitúa por debajo.

- viAs: Variable dummy, que representa el tipo de vía. Toma valor 1 cuando las vías son carreteras nacionales o autopistas urbanas o comarcales, y 0 cuando las vías son urbanas o comarcales.

- REFINO: Variable dummy, que toma valor 1 cuando en la provincia existen instalaciones de refino y almacenamiento.

\section{c) Resultado}

En este apartado se presentan los resultados del modelo empírico propuesto. Éstos se resumen en el cuadro 1, donde se presentan dos contrastes. El primero incluye únicamente las principales variables explicativas, en línea con las proposiciones teóricas que se quieren contrastar, y en el segundo se incluyen las variables que controlan el efecto de los otros factores que pueden afectar la estructura competitiva del sector

Uno de los resultados del modelo teórico propuesto anteriormente constata que un incremento en el nivel mínimo de ingresos en la región tiene un impacto muy positivo sobre los de las estaciones de servicio abanderadas (proposición 1). Por tanto, en aquellas regiones donde el nivel mínimo de ingresos es alto existe una mayor probabilidad de que predominen estaciones de servicio abanderadas con una marca, ya que en estas condiciones de mercado se favorece la demanda por un producto de alta calidad, en detrimento del de baja calidad. El incremento del poder adquisitivo de los consumidores, y particularmente de los de menores ingresos de la región, se puede traducir en un cambio de sus preferencias por la calidad, que se alinean a favor de consumir aquel producto que puede ser percibido como de mayor calidad. Así, es de esperar que el coeficiente $\beta_{1}$ de la variable MININGRESO sea positivo.

Se comprueba que aquellas regiones en las cuales los niveles de renta característicos más bajos son altos, la probabilidad de encontrar estaciones de servicio abanderadas con marcas reconocidas es mayor $\beta_{1}=0.113>0$. Este resultado, expresado en términos de elasticidades, indica que el efecto marginal del incremento en un punto en el índice que mide el nivel de ingresos mínimo de la región donde opera la estación de servicio es de 0.022 , teniendo en cuenta que el estimador de la probabilidad es para el valor medio. Una posible explicación a estos resultados la encontramos en la teoría de los costos de búsqueda. En merca- 
dos con mayor poder adquisitivo, los consumidores se caracterizan por tener altos costos de búsqueda y, por tanto, una mayor predisposición a pagar por los productos de marca; según dejan constancia PNG y Reitman (1994), ésta constituye una señal fiable y creíble de la calidad de los productos.

En el apartado teórico se ponía de manifiesto el impacto de la densidad de los consumidores sobre la demanda y los beneficios de las empresas. De acuerdo con la proposición 2, en la medida que la demanda de consumidores se incrementa, una estación de servicio se beneficia en mayor medida si se abandera con una marca de reconocido prestigio. Por tanto, a priori podría ser más probable que, en un mercado con altos niveles de densidad de población, una gasolinera se beneficie operando bajo el amparo de una marca de reconocido prestigio.

Los resultados del cuadro 1 indican que efectivamente en la medida que aumente la densidad de población, la probabilidad de que una estación de servicio se abandere con la marca de una refinería de reconocido prestigio es mayor, ya que $\beta_{2}=0.173>0$. Esto da señal de que en mercados más concentrados demográficamente la estrategia contractual de una estación de servicio estaría más orientada a diferenciarse verticalmente operando con una marca reconocida, que a centrar su estrategia en competir más agresivamente en precios.

CUADro 1. Resultados del modelo Logit. Variable dependiente BRAND*, **

\begin{tabular}{|l|r|c|c|c|}
\hline \multicolumn{1}{|c|}{ Variable } & Coeficiente & Estadístico Wald & \multicolumn{1}{c|}{ Sig. } & Exp $(B)$ \\
\hline MININGRESOS & 0.113 & 4.890 & 0.026 & 1.461 \\
\hline DENSCONSUM & 0.173 & 4.320 & 0.031 & 1.175 \\
\hline DENSVEHICULOS & 0.362 & 12.353 & 0.000 & 1.479 \\
\hline INTENSIDAD & 0.420 & 4.711 & 0.030 & 1.541 \\
\hline VIAS & 0.370 & 11.145 & 0.001 & 1.412 \\
\hline REFINO & 0.553 & 8.645 & 0.003 & 1.792 \\
\hline N & 4.164 & & & \\
\hline-2 Log de la verosimilitud & $2,307.509$ & & & \\
\hline$R$ cuadrado de Cox y Snell & 0.435 & & & \\
\hline$R$ cuadrado de Nagerkelke & 0.632 & & & \\
\hline
\end{tabular}

* El modelo cumple las condiciones de normalidad de los residuos-Kolmogorov Smirnoff $Z=21.98($ Sign $=0.00)$.

** La robustez del modelo se ha comprobado tras superar las pruebas de Leverage (0.028), DFBetas $(<0.05)$ y distancias de Cook.
Por último, en cuanto a las variables de control que hemos incorporado para recoger el impacto de la intensidad de la competencia y de la localización, en la probabilidad de ocurrencia de contratos, los resultados se alinean con los esperados y citados en la bibliografía. En primer lugar, la variable INTENSIDAD, que recoge el posible efecto de la intensidad competitiva medida como el número de estaciones de servicio por unidad de superficie, tiene un impacto positivo y estadísticamente significativo en la probabilidad de que una estación de servicio opere abanderada con una marca reconocida. Netz y Taylor (2002) afirman que en el mercado estadounidense las estaciones abanderadas con marcas de renombre tienen incentivos para maximizar la diferenciación espacial en presencia de estaciones independientes con el objetivo de minimizar la intensidad competitiva Sin embargo, según los resultados expuestos, en el mercado español la estrategia competitiva llevada a cabo por los empresarios de estaciones de servicio indica que en la medida en que la intensidad competitiva aumenta, las estaciones de servicio optan por una estrategia de diferenciación para rentabilizar el negocio.

El coeficiente de la variable VIAS indica que es más probable la existencia de estaciones de servicio abanderadas en carreteras nacionales y autopistas. La explicación de este resultado la encontramos en el hecho de que, como hemos señalado anteriormente, en este tipo de vías ocurren condiciones competitivas singulares, debido a que son vías rápidas y los automovilistas tienen menos incentivos para detenerse a recargar. Por tanto, es factible que operando con una marca reconocida se pueda aumentar el atractivo de la estación de servicio y contrarrestar así el desincentivo a las recargas de los viajantes.

Por último se constata que la existencia de instalaciones de refino y logísticas (CLH) en la región tiene un efecto importante en la estructura competitiva del sector, ya que en tales condiciones parece evidente que la existencia de menores costos logísticos permite al empresario favorecerse con precios más competitivos, y además aprovechar las bondades de operar con esa marca reconocida en esa región.

\section{Conclusiones}

El sector español de distribución minorista de carburantes viene experimentando cambios importantes en su estructura a partir del proceso de apertura y liberalización del mercado iniciado en 1992. El ingreso de nuevos operadores al mercado y sobre todo el aumento de estaciones de servicio independientes han dado origen a una oferta más diversificada que posibilita a los consumidores la elección de distintas alternativas de producto. 
En el sector de distribución minorista de carburantes en estaciones de servicio encontramos, en función del tipo de contrato que las liga con sus suministradores, estaciones que operan abanderadas con marcas reconocidas y también estaciones independientes. En este trabajo hemos realizado un estudio del impacto que las características de la demanda puede tener en la estructura competitiva del sector. En el contexto actual, el estudio de estos aspectos cobra especial relevancia ya que los empresarios de estaciones de servicio han adquirido mayor flexibilidad y poder de decisión a la hora de negociar sus contratos con las grandes las refinerías, y pueden optar por distintas formas contractuales que las liguen a éstas.

La principal contribución del modelo teórico propuesto es que los niveles de renta y la densidad de población tienen efectos importantes en la demanda y los beneficios de las empresas. Los niveles de renta característicos afectan positivamente a la demanda y los beneficios de las empresas, y en mayor medida a las empresas que ofrecen un producto de alta calidad. El efecto de la densidad de población es similar. Estos resultados han sido contrastados utilizando una muestra de 4,164 estaciones de servicio donde están representadas todas las comunidades autónomas de España.

Los resultados empíricos aplicados al mercado español arrojan resultados interesantes para los empresarios de estaciones de servicio, ya que aportan información relevante sobre la estructura competitiva del sector en relación con las características socioeconómicas y la intensidad competitiva en las distintas provincias españolas. Los resultados ponen de manifiesto que efectivamente la demanda y los beneficios de las empresas se verán afectados por las características de los mercados en los que operen. Los indicadores socioeconómicos de estas regiones determinan el tipo de estación que opera en el mercado final y, por tanto, deberían de tenerse en cuenta para decidir el tipo de contrato más adecuado en la región en la cual se encuentre la estación de servicio.

En aquellas regiones con mayores niveles de renta se constata una mayor probabilidad de presencia de estaciones abanderadas con marcas reconocidas que de estaciones independientes. A medida que aumenta la renta mínima de la región, la probabilidad de una mayor presencia de estaciones de servicio abanderadas aumenta. Además, la concentración demográfica es otro factor determinante para explicar la estructura competitiva del sector. Cuanto mayor sea la densidad demográfica, aumenta la probabilidad de encontrar estaciones de servicio abanderadas con marcas reconocidas. En estas situaciones, la estrategia de diferenciación por la marca se impone a una estrategia centrada en la reducción de costos, ofreciendo un producto sin marca en estaciones independientes.
Las principales limitaciones del trabajo provienen principalmente de los datos utilizados. En primer lugar, el carácter transversal de los datos impide realizar afirmaciones definitivas sobre las relaciones de causalidad que existen entre las variables. Además si bien la muestra de estaciones de servicio es amplia y abarca todo el territorio español, desafortunadamente no hemos contado con más información específica de cada estación en aspectos relacionados al nivel de servicio ofrecido, tipo de contrato respecto a la propiedad y gestión, así como condiciones de competencia local (en un radio de $2 \mathrm{~km}$ alrededor de cada estación de servicio). Actualmente, la información disponible sobre la red no permite abordar estos aspectos; no obstante, en futuras investigaciones se intentará aportar este tipo de información para dar así aún mayor consistencia a los resultados que se ofrecen en este trabajo.

\section{REFERENCIAS BIBLIOGRÁFICAS}

Barron, M. (2004), "Number of Sellers, Average Prices, and Price Dispersion". International Journal of Industrial Organization 22: 1041-1066.

_ B. Taylor, y M. Umbeck, (2000), "A Theory of Quality Related Differences in Retail Margins: Why There Is a 'Premium' on Premium Gasoline”. Economic Enquiry 38 (4): 550-569.

— y M. Umbeck, (1984), "The Effect of Different Contractual Arrangements: The Case of Retail Gasoline Markets". The Journal of Law and Economics 17: pp. 313-328.

Bello, A., y S. Cavero (2007 a), "Estructura y estrategia competitiva en el mercado español de carburantes". Economía Industrial 350: 97-112.

—_ (2007 b), "Competencia estratégica en la distribución minorista de carburantes y combustibles de automoción en España". Revista de Economía Aplicada 45: 125-154.

_ , (2008), "The Spanish Retail Gasoline Market. New Patterns of Competition since the Liberalization of the Industry". Energy Policy 36:612-626.

Blass, A., y D. Carlton (2001), "The Choice of Organizational Form in Gasoline Retailing and the Cost of Laws Limiting That Choice". Journal of Law and Economics XLIV: 511-524.

Borenstein, S. (1991), "Selling Costs and Switching Costs: Explaining Retail Gasoline Margins". Rand Journal of Economic 22: 345-369.

Comanor, W., y J. Riddle (2003), "The Costs of Regulations: Branded Open Supply and Uniform Pricing of Gasoline". International Journal of the Economics of Business 10: 135-55. 
Eckert, A., y W. Douglas (2005), "Price Uniformity and Competition in a Retail Gasoline Market”. Journal of Economic Behavior and Organization 56: 219-237.

Enciclopedia del petróleo, petroquímica y gas. Años: 1995 a 2006. Madrid: oILGAS.

Guía de estaciones de servicio, proveedores y operadores (2003), núm. 169. Madrid: Tecni-Publicaciones.

Hastings, J. (2004), "Vertical Relationships and Competition in Retail Gasoline Markets Empirical Evidence from Contract Changes in Southern California". The American Economics Review 94 (1): pp. 317-328.

Iyer, G., y P. Seetharaman (2003), "To Price Discriminate or Not: Product Choice and The Selection Bias Problem”. Quantitative Marketing and Economics 1: pp. 155-178.

Meerbeeck (2003), "Competition and Local Market Conditions on the Belgian Retail Gasoline Market". The Economist 151 (4): pp. 369-388.

Mills, D. E. (1995), "Why Retailers Sell Private Labels". Journal of Economics and Management Strategy 4: pp. 509-528.

Netz, S., y B. Taylor (2002), "Maximum or Minimum Diferentiation? Location Patterns of Retail Outlets". The Review of Economics and Statistics 84 (1): pp. 162-175.

PNG, I., y D. Reitman (1995), "Service Time Competition". Rand Journal of Economics 25: pp. 619-634

Segrelles (2003), www.aop.es

Slade, M. (1993), "Strategic Motives for Vertical Separation: An Empirical Exploration". Journal of Law, Economics, and Organization 14 (1): pp. 84-113.

Spengler, J. (1950), "Vertical Integration and Antitrust Policy". Journal of Political Economy 58: pp. 347-352.

Vita, M. (2000), "Regulatory Restriction on Vertical Integration and Control: The Competitive Impact of Gasoline Divorcement Policies". Journal of Regulatory Economic 18 (3): pp. 217-233.

Williamson, O. E. (1975), Markets and Hierarchies. Nueva York: Free Press.

\section{¿Es estable la relación entre tipo de cambio y posiciones netas de los especuladores en monedas de economías desarrolladas? Evidencia del Chicago Mercantile Exchange}

Leonardo Egidio Torre Cepeda* y María Elena Lavín Morales**

\section{RESUMEN}

Este trabajo analiza si la relación entre los cambios en las posiciones netas de los especuladores de monedas de cinco economías desarrolladas en el Chicago Mercantile Exchange y los cambios porcentuales semanales en los tipos de cambio con respecto al dólar estadounidense de dichas monedas es estable en el tiempo, o bien depende, en alguna medida, del tamaño del mercado para los contratos de futuros de dichas monedas. Los resultados sugieren que no puede rechazarse la hipótesis de que el crecimiento del mercado incide sobre la sensibilidad del tipo de cambio ante una variación en las posiciones netas de los especuladores.

Número de clasificación: JEL: F31, F32, F39.

Palabras clave: tipo de cambio, posiciones netas de especuladores, enfoque de microestructura, flujo de órdenes, mercado de futuros.

\section{Abstract}

This paper analyzes whether the relationship between the changes in the net position of noncommercial traders in the currencies of five developed economies at the Chicago Mercantile Exchange, and the percentage changes in the exchange rates - relative to the us dollar- of such currencies is time invariant, or whether it depends upon the size of the corresponding currency futures market. Our results show that, for the period analyzed, the relationship is unstable in all cases, finding that it can not be discarded as being connected to market size growth.

Number of classification: JEL: F31, F32, F39.

Key words: exchange rate, net positions of speculators, microstructure approach, order flows, futures market.

Profesor de tiempo completo en la Facultad de Economía de la UANL [ltorre@ faeco.uanl.mx y leonardoegidio.torre@cemex.com].

Coordinadora de información financiera de Supermercados Internacionales H-E-B, [mlavin@ hebmex.com].

Los autores agradecen a los participantes en el Seminario de Organización Industrial de la Facultad de Economía de la UANL, al Comité Nacional de Estudios Económicos del IMFF, así como dos dictaminadores anónimos por sus valiosos comentarios. Todos los errores en el documento, sin embargo, son responsabilidad exclusiva de los autores. 